\title{
Q fever outbreak in Cheltenham, United Kingdom, in 2007 and the use of dispersion modelling to investigate the possibility of airborne spread
}

\author{
A Wallensten ${ }^{1,2}$, P Moore ${ }^{3}$, H Webster ${ }^{4}$, C Johnson $^{1}$, G van der Burgt ${ }^{5}$, G Pritchard ${ }^{6}$, J Ellis-Iversen ${ }^{7}$, I Oliver \\ (isabel.oliver@hpa.org.uk)1,8 \\ 1. Health Protection Agency South West, Stonehouse, United Kingdom \\ 2. European Programme for Intervention Epidemiology Training (EPIET), European Centre for Disease Prevention and Control, \\ Stockholm, Sweden \\ 3. Gloucestershire Hospitals NHS Foundation Trust, United Kingdom \\ 4. Met Office, Exeter, United Kingdom \\ 5. Veterinary Laboratories Agency, Luddington, United Kingdom \\ 6. Veterinary Laboratories Agency, Bury St Edmunds, United Kingdom \\ 7. Centre for Epidemiology and Risk Analysis, Veterinary Laboratories Agency, Weybridge, United Kingdom \\ 8. Bristol University, Department of Social Medicine, Bristol, United Kingdom
}

Citation style for this article: Wallensten A, Moore P, Webster H, Johnson C, van der Burgt G, Pritchard G, Ellis-Iversen J, Oliver I. Q fever outbreak in Cheltenham, United Kingdom, in 2007 and the use of dispersion modelling to investigate the possibility of airborne spread . Euro Surveill. 2010;15(12):pii=19521. Available

online: http://www.eurosurveillance.org/ViewArticle.aspx?Articleld=19521

We describe the investigation of an outbreak of $Q$ fever in the town of Cheltenham, England. The outbreak was detected in June $\mathbf{2 0 0 7}$, and prospective and retrospective case finding identified 30 confirmed or probable human cases. The investigation identified windborne spread of Coxiella burnetii from nearby sheep farms as the most likely source of infection. A telephone survey was conducted to identify risk practices at local farms. Subsequently the atmospheric dispersion model NAME was used to identify whether air from the identified farms with high risk practices had been carried into Cheltenham town centre during the risk period. Three high risk farms were identified and the modelling showed that air from all of these farms was carried over Cheltenham in the estimated risk period. The investigation resulted in an information campaign to farmers and production of improved advice for livestock farmers on reducing the risks of transmitting $Q$ fever to humans.

\section{Introduction}

Q fever is caused by the bacterium Coxiella burnetii, which has major zoonotic potential and is found worldwide in many different animal species, including wildlife [1]. It is not a notifiable disease in animals or humans in the United Kingdom (UK). Infection in animals is mainly subclinical and inapparent, although it can occasionally cause abortion. However, specific laboratory examinations for $C$. burnetii are not undertaken routinely in animals in the UK, and the infection is only likely to be detected as part of in-depth investigations into major abortion outbreaks in domesticated ruminant species. Furthermore, the veterinary diagnostic tests that are currently available are of limited value. Hence accurate surveillance data on prevalence is lacking, although it is considered endemic in domestic animal populations [2-3]. Large numbers of bacteria are present in the placenta and birth products of infected animals and are released during delivery [1]. The bacterium persists in the environment in a resistant spore-like form which may become airborne and transported long distances by the wind [4-7].

Humans may contract disease by inhalation or, more rarely, by drinking unpasteurised milk or through tick bites. The incubation period varies from 10 to 14 days but may be as long as 39 days depending on the infectious dose [8]. Disease in humans ranges from asymptomatic to severe and can be fatal. It often presents with fever or influenza-like illness, but may cause pneumonia, hepatitis, meningoencephalitis or perimyocarditis. Rarely the disease becomes chronic and leads to endocarditis [9]. Infection, particularly early in pregnancy, may result in abortion, or later in pregnancy to premature labour [10]. Several human outbreaks of $Q$ fever have occurred in the UK, some of which have been associated with windborne spread of contaminated material from infected animals and contaminated farmland [5,11-12].

On 29 June 2007, the Gloucestershire Health Protection Team was notified of five cases of $Q$ fever in patients living in the town of Cheltenham, England with onset of illness between 1 May and 14 June. No cases had been reported in this area in the previous three years. An outbreak investigation team was summoned to investigate the outbreak, consisting of members of the Health Protection Agency South West (HPA SW), Gloucestershire Hospitals National Health System (NHS) Foundation Trust, Cheltenham Borough Council, 
HPA Centre for Infections (CFI), Veterinary Laboratories Agency (VLA) and Defra. It was considered that airborne infection from infected animals was the most likely source of the outbreak and the investigations described here were subsequently initiated to pursue this hypothesis further.

\section{Methods}

Epidemiological and laboratory investigations

Suspected cases were interviewed by telephone by staff of the HPA SW using a standard questionnaire for $Q$ fever with additional questions relevant to the local area. The questionnaire included questions on: animal exposure, food history (including consumption of unpasteurised milk), risk activities, work details, tick exposure, distance of home to farmland and places visited. After a 6th case was reported, it was decided that active case finding should be undertaken. All General Practitioners (GPs) in the Cheltenham area were informed of the outbreak and encouraged to consider the diagnosis of $Q$ fever in patients with relevant symptoms. Retrospective case finding was attempted by investigating hospital admission records for diagnoses of unspecified pneumonia. The purpose was to identify additional patients whose exposure history may have helped to identify a source of infection. No attempt was made to identify all possibly infected patients in the county as it was felt that self-limiting illnesses did not need to be identified. The most common presenting clinical feature that could be distinguished by clinical coding of admissions was searched for. Patients discharged with a diagnosis of unspecified pneumonia were contacted and asked to leave a blood sample for $Q$ fever serology.

The standard laboratory method in use in the clinical diagnostic laboratory for screening for $Q$ fever was the phase I and II complement fixation test (CFT) which, if positive at a titre of 1:16 or greater, prompted referral of the serum to the HPA reference laboratory for analysis by enzyme-linked immunoassay (EIA).

\section{FIGURE 1}

Epidemic curve for the outbreak of Q fever in Cheltenham 1 May-8 July $2007(n=30)$

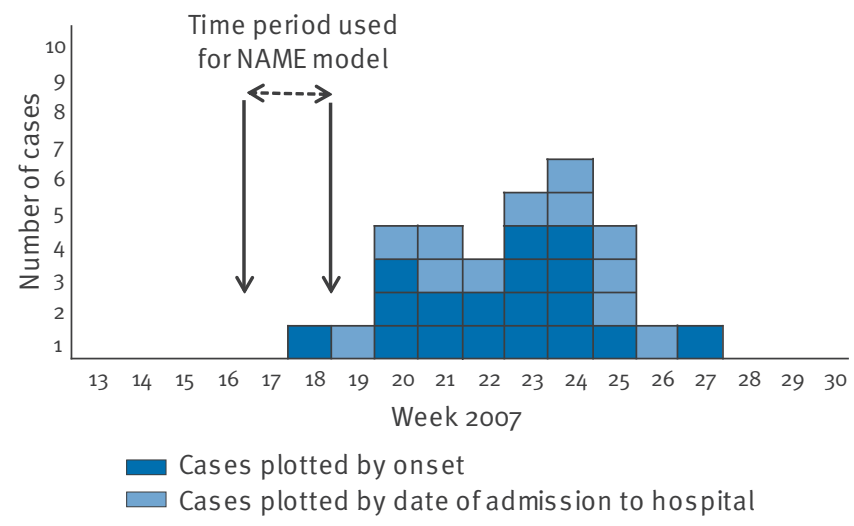

Cases were plotted with date of onset if this information was known, otherwise the date of hospital admission was used.
The following case definition was used in the investigation: A confirmed case was defined as a person who lived in or visited Cheltenham between 1 April and 31 June 2007 who presented with pneumonia or clinical symptoms consistent with $Q$ fever acquired during this time period. A confirmed case subsequently also needed to exhibit IgM antibody titres displaying assay positivity in serial dilutions of more than 1:80 or a four-fold increase of phase II CFT titres against C. burnetii with paired sera taken at least seven days apart. A probable case was defined as a person that lived in or visited Cheltenham between 1 April and 31 June 2007 who presented with pneumonia or clinical symptoms consistent with $Q$ fever acquired during this time period. A probable case subsequently also needed to exhibit IgG antibody titres displaying assay positivity in serial dilutions of more than 1:80 or a single test with four-fold raised CFT titre against $C$. burnetii compared to baseline. The risk period for exposure was at the time of the ongoing outbreak investigation estimated to have been 23 April-7 May 2007. This period was chosen to cover the time distribution of cases from the earliest disease onset date minus maximum incubation period until onset of disease in the last detected case minus minimum incubation time. More cases were identified retrospectively. Testing for $Q$ fever was initiated by GPs or hospital physicians on request of the Health Protection Agency South West on the basis of respiratory symptoms or symptoms of a influenza-like illness (such as malaise, lethargy, myalgia, arthralgia and headache). Additionally, blood samples received for testing for any respiratory pathogens were automatically included by the laboratory.

\section{Veterinary investigation}

The location of livestock farms in the vicinity of Cheltenham and the numbers of animals present were mapped using data obtained from the Animal Health Agency. The VLA was consulted regarding recent reports of $Q$ fever affecting farm animals in the area. In order to further investigate the possibility that nearby livestock farms may have been the source of the outbreak, a semi-structured telephone questionnaire survey was carried out to investigate any potentially relevant disease history (i.e. abortions/reproductive failures) and husbandry practises on local farms that may have contributed to the risk. The selection of farms was based on their geographical location in relation to the distribution of cases, using meteorological information regarding the predominant wind directions at the likely time period of exposure. The likelihood of a farm being the source of the outbreak was categorised empirically using a qualitative risk assessment approach, where three categories of risk factors were assessed. Firstly, the risk of infection: human illness, reproductive problems in livestock and presence of ticks that are potential vectors for $C$. burnetii. Secondly, the risk of release: lambing/calving dates and place, manure handling and movement of animals. Lastly the risk of human contact: distance from town centre, distribution of manure 
to public, transport of animals and manure in densely populated areas and public access to animals. The qualitative risk assessment was based on the proportion of risk practices present in relation to all possible risk practices. Since little is known about the relative importance of different risk factors for transmission of $C$. burnetii in farm animals, the practices were not weighted. The farms were categorised as low, medium or high risk, based exclusively on the risk assessment, without establishing whether $C$. burnetii was present in the animals.

\section{Environmental investigation}

In addition to livestock farms, other premises with livestock such as abattoirs and livestock markets were considered as possible sources. Information on these was obtained via the Meat Hygiene Service and local Animal Health offices. Information on other events involving animals which had been held in the area and the location of allotments (potential manure risk) were also collected. Risk sites were visited and investigated.

\section{Meteorological investigation}

Meteorological observations of near surface (10 m above ground) wind speed and wind direction in the time period before the onset of disease in confirmed and probable cases were obtained from the Met Office's observation site in Pershore approximately 30 $\mathrm{km}$ north of Cheltenham to help identify the source of the outbreak. This information was used to assist the veterinary investigation to decide on which farms to interview as described above.

Later, Numerical Atmospheric-dispersion Modelling Environment (NAME) [13], an atmospheric dispersion model, was used to investigate potential airborne transport of the $C$. burnetii between a number of suspected sources (notably local farms identified by veterinary investigation) and the infected persons in Cheltenham. NAME has a wide range of applications including air quality forecasting, predicting the transport and spread of chemical, biological and nuclear material, producing volcanic ash forecasts, identifying source locations and strengths, investigating pollution episodes and airborne spread of diseases. The model can be run in forward mode, predicting the transport and spread of airborne material released from an identified source. Alternatively, it can be run in backward mode, predicting the transport backwards in time from an identified receptor point, thereby showing the air history of material arriving at the receptor point and identifying potential sources.

NAME was run for the estimated risk period 23 April to 7 May, both in forward mode to give the predicted area at risk from the suspected farms and in backward mode to give the air history for air arriving in Cheltenham. Input meteorological data used in this study to drive NAME was hourly three-dimensional meteorological data from the Met Office's numerical weather prediction model (the Unified Model [14]) with a horizontal spatial resolution of $12 \mathrm{~km}$. The accuracy of the atmospheric dispersion modelling is directly related to the accuracy of the input meteorological data and, whilst the meteorological data is likely to represent the larger scale atmospheric motions, it is not expected to capture the small scale local flow within the urban conurbation of Cheltenham (e.g. channelling of the flow within street canyons).

\section{TABLE}

Patient demographics and presenting symptoms, Q fever in Cheltenham May-July 2007 (n=30)

\begin{tabular}{|l|l|}
\hline Criteria & Number (\%) \\
\hline Age range & $19-72$ years \\
\hline Male & $21(70 \%)$ \\
\hline Female & $9(30 \%)$ \\
\hline Hospital admission & $24(80 \%)$ \\
\hline Identified retrospectively & $15(50 \%)$ \\
\hline Smoker & 11 current smokers (37\%), 7 ex-smokers (23\%) \\
\hline Non-smoker & $9(30 \%)$ \\
\hline Smoking status unknown & $3(10 \%)$ \\
\hline Presenting symptoms: & \\
\hline Fever & $25(83 \%)$ \\
\hline Headache & $17(57 \%)$ \\
\hline Myalgia/Arthralgia & $18(60 \%)$ \\
\hline Chest pain & $13(43 \%)$ \\
\hline Cough/Shortness of breath ${ }^{1}$ & $26(87 \%), 13 / 15$ non lookback (87\%) \\
\hline Nausea (N), vomiting $(V)$, diarrhoea (D) & 4 NVD, 3 NV, 2 N, 1 D \\
\hline Other symptoms & 2 Loin pain, 2 dizziness, 1 confusion, 1 skin rash \\
\hline
\end{tabular}

1 Because retrospective case finding was based on presentation with pneumonia, the proportion with cough that were not part of retrospective case finding is also shown. 


\section{Results}

Descriptive epidemiology and

laboratory findings

Interviews with suspected and confirmed cases did not reveal any common exposures other than living in or having visited Cheltenham town centre. The questionnaire did not identify any shared risk exposures or activities that could have resulted in transmission. The only factor the cases had in common was being a resident of or having visited central Cheltenham. A total of 30 cases all living in Gloucestershire were identified that met the case definition for a confirmed or probable case in the outbreak period. Fifteen had been identified through retrospective case finding among people

hospitalised with pneumonia. Of the total of 30 cases, nine were female and 21 were male. The age range was 19-72 years and the median age was 48 years. The first onset of disease was on 1 May and the last on 8 July (Figure 1). Twenty-four cases were hospitalised. A summary of reported symptoms and demographics is given in the Table.

\section{Veterinary investigation}

There had been no recent reports of $Q$ fever affecting farm animals in the area. We identified sixteen

\section{FIGURE 2}

NAME air dosage maps obtained by modelling a continuous release from the high risk farms (A, B, and C) for the time period 23 April-7 May 2007
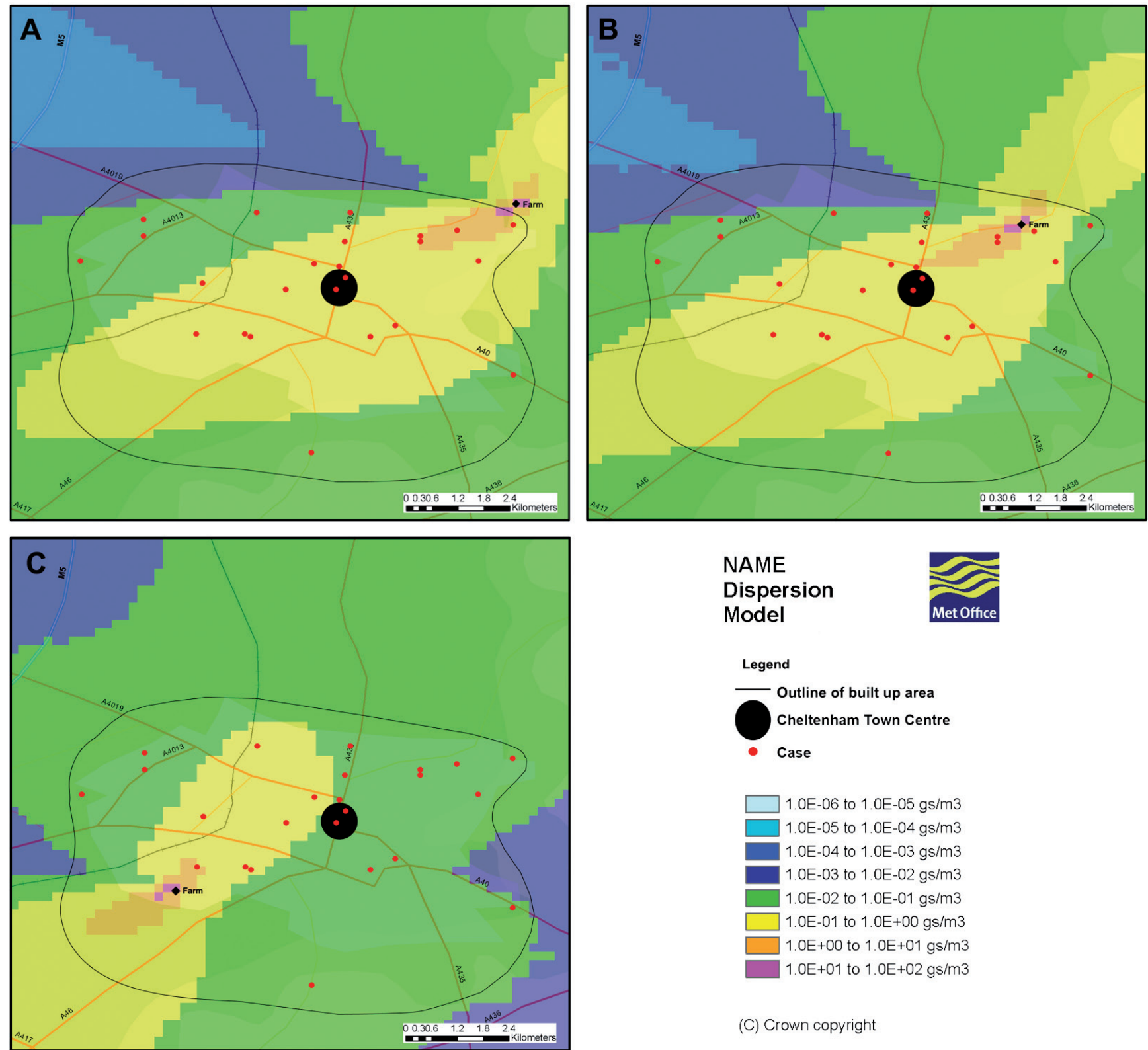

\section{NAME \\ Dispersion \\ Model}
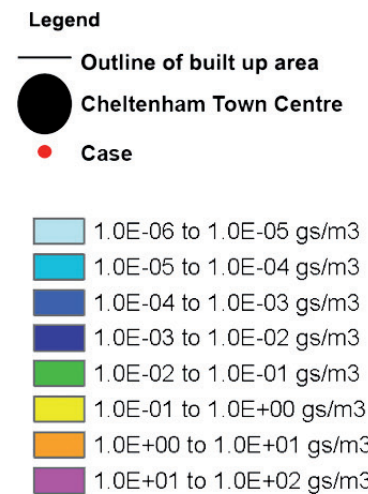

(C) Crown copyright

NAME: Numerical Atmospheric-dispersion Modelling Environment.

The areas of highest dosage (pink, orange and yellow) covers Cheltenham town centre. A black diamond marks the location of the farm. Red dots mark the addresses of cases resident in Cheltenham. A black line illustrates the outer limit of the built up areas in Cheltenham. The filled black circle marks the town centre which all cases, including those not resident in Cheltenham, had visited at some time during the risk period for exposure. 
farms that stocked farm animals in proximity to the Cheltenham town centre that were located along a south-west to north-easterly line to accommodate for the predominant wind directions for the period from 23 April to 7 May. Eleven farms completed the telephone survey. The remaining five farms either did no longer have livestock or could not be contacted. The risk assessment classified five low-risk farms, three medium-risk farms and three high-risk farms. The high risk farms $A, B$, and $C$ were located $4.4 \mathrm{~km}, 2.7 \mathrm{~km}$ and $4.2 \mathrm{~km}$ from the town centre. All three farms stocked sheep and two farms also stocked cattle. Farm A calved and lambed during the risk period, transported a large batch of animals through Cheltenham town centre and sold manure to nearby allotments. Farm B reported outdoor lambing during the risk period and burning the fresh straw bedding and birth products outside every few days. This is an unusual management practice. Usually, abortion and birth products would be incinerated and bedding stacked up in a heap for a long time to kill pathogens before disposal. Farm C reported outdoor lambing and a few stillbirths and had several sheep movements close to the town centre.

\section{Environmental investigation}

The environmental investigation did not reveal any places or events in the town of Cheltenham that could have posed a risk during the estimated risk period from 23 April to 7 May.

\section{Meteorological investigation}

According to the meteorological observations from the Pershore observation site the predominant wind direction in the Cheltenham area during the estimated risk period was from the north-east and to a lesser extent from the south-west, which is the prevailing wind direction in the UK. Wind speeds were unexceptional, ranging from light winds to breezy conditions. Dispersion modelling using NAME showed that air from each of the suspected farms may have exposed the town to the bacterium at some point over the study period assuming there had been a continuous release (Figure 2). Therefore, none of the suspected farms could be ruled out as potential sources.

\section{Discussion}

At least 30 people were infected with $C$. burnetii in this outbreak. Further cases were not sought from household contacts or those with other possible presenting symptoms as the aim of the retrospective case finding was to aid the epidemiological investigation to identify the source of the outbreak. Previous outbreaks indicated that $2 \%-5 \%$ of those infected may be hospitalised [15]. Extrapolating from the 15 cases we identified retrospectively through hospital admission suggests that possibly up to 500 people may have been infected. The population of Cheltenham town is approximately 110,000 people and there are approximately 560,000 people in Gloucestershire who may visit Cheltenham as well as possible visitors from outside the county. National guidelines do not recommend the identification of all patients with $Q$ fever and the seroprevalence among farmers, veterinarians, and people living in rural communities suggest that undiagnosed infection is common [15-16]. It was therefore not deemed appropriate to attempt mass screening.

The age and sex distribution of identified cases was similar to that of other outbreaks [1]. The epidemic curve shows that cases fell ill over a period of at least seven weeks, suggesting either that the release of bacterium was continuous or intermittent over a similar number of weeks or that the incubation period varied greatly. The incubation period may be prolonged when the infectious dose is small, which is likely in long-distance windborne transmission. No common risk factor was identified between cases other than living in, or having visited, Cheltenham and therefore we hypothesised that windborne spread of $C$. burnetii from nearby farms was the probable source of infection. Conditions were at times breezy, and strong winds have played a role in other outbreaks [5]. The predominant wind direction during the two-week period studied was from the north-east rather than the prevailing wind direction from the south-west which was the second most common wind direction. The telephone survey to the selected group of nearby farms revealed some high risk practices that could potentially have resulted in windborne spread. These were discussed with the farmers concerned and the practices ceased. Advisory information for farmers on $\mathrm{Q}$ fever control was also circulated via veterinary practices in England and Wales and put on VLA, HPA and HSE websites. Transportation of animals through populated areas has caused outbreaks previously [17], as have outdoor lambings [7]. One farm burnt the fresh straw bedding and birth products outside on several occasions, and this practice may facilitate windborne spread of $C$. burnetii by releasing incompletely burnt contaminated material into the air. The number of farms contacted was fairly small because of limited resources, but it included all the main livestock farms in the area. However, the possibility that we missed other farms with risk practices cannot be ruled out.

Laboratory investigation of the animals on the highrisk farms for evidence of C.burnetii infection was considered by the outbreak control team but was decided against, because the potential value of any results was perceived to be limited. The long time interval from the exposure date to sampling of the animals would complicate interpretation because, for example, farms may have sold infected animals that had aborted. Furthermore, interpretation of positive results would be complicated by the fact that little is known about the seroprevalence of infection in livestock in the UK generally and it would not be possible to put the serological results into perspective. The only scientifically viable option would have been to design a prevalence study combined with collection of risk factor information on all farms in the area. That was considered beyond the scope of this outbreak investigation. 
To further support the outbreak investigation hypothesis that windborne spread from a local farm caused the cases in Cheltenham we employed the use of an atmospheric dispersion model. We chose NAME as this model has previously been used successfully to investigate airborne spread of diseases such as foot and mouth [18], bluetongue [19] and Legionnaires' disease. The model was run for the period from 23 April to 7 May. This time period was chosen as it would have explained the cases that were known at the time of the outbreak investigation but cannot explain all cases that were identified later. The modelling showed that air from all the farms was transported to Cheltenham town centre at some point during the period studied. Each of them could therefore have been the potential source of infection and none of the high-risk farms could be excluded. As we do not know the exact dates of transmission, we cannot say that one farm was more likely than the other, as the wind directions may have varied day by day within the studied period.

The modelling could, however, have been refined to potentially give more conclusive evidence, if further detailed information regarding the outbreak had been obtained such as more specific information on the potential time of release of $C$. burnetii, release rates of the bacterium, concentrations required for infection and exact time of infection. These parameters were not available due to uncertainties in the epidemiological investigation. Firstly, we know that all the cases lived in or visited Cheltenham town centre, but we do not know whether their presence coincided in time with windborne transport of contaminated air to Cheltenham town centre from a high risk farm. Secondly, the area of risk of exposure calculated by the atmospheric dispersion model may be an overestimate, if the release of $C$. burnetii was not continuous over the two-week transmission period identified. The release may not have been continuous as the time period between disease onsets suggests that people were infected intermittently over an extended period. Thirdly, although C. burnetii infection is considered endemic in UK farm animals, precise information about the infectious status of the investigated farms was unavailable. A well structured serological survey to measure the extent of C. burnetii infection in farmed livestock in the UK would answer questions relating to prevalence and relative geographical risk to the human population and greatly assist any further similar outbreak investigations.

\section{Conclusion}

Despite limitations, we believe that atmospheric dispersion models can be a valuable tool in similar outbreak investigations and this is supported by other disease outbreak studies using NAME [18-19]. In the Cheltenham outbreak it added support to our hypothesis of windborne spread of $C$. burnetii from a high risk farm, when an analytical study was not feasible. Furthermore, this investigation identified likely risk practices on local farms and engaged concerned stakeholders in the consideration of preventive measures leading to improved advice for farmers [20]. Enhanced local surveillance in the area in the following year (2008) did not reveal any cases of $Q$ fever which suggests that high risk practices may have ceased.

Finally this investigation showed the strength and benefits of different agencies and authorities working closely together. In this investigation the close collaboration and information exchange between veterinary, human health, and meteorological agencies and the local authorities was perceived as beneficial by all involved. We believe that sharing and applying different techniques and information between different fields of research is of paramount importance for successful outbreak investigations.

\section{Acknowledgements}

We would like to thank the following people for their assistance in the investigation: Leo Foyle and Richard Smith at the Veterinary Laboratories Agency, Andrew Frost and Harry Bailie at DEFRA, Robert Smith at the National Public Health Service for Wales, Deirdre Lewis, Andrea Penketh, Adrienne Hegarty, Stephen Palmer, Stephen Edwardson, Dilys Morgan, Mandy Walsh, Laurence Knight, Rebecca Close and Chitra Arumugam at the Health Protection Agency as well as staff at the HPA Laboratory.

\section{References}

1. Maurin M, Raoult D. Q fever. Clin Microbiol Rev. 1999;12(4):518-53.

2. Paiba GA, Green LE, Lloyd G, Patel D, Morgan KL. Prevalence of antibodies to Coxiella burnetii (Q fever) in bulk tank milk in England and Wales. Vet Rec. 1999;144(19):519-22.

3. Defra. Zoonoses Report United Kingdom 2006. Available from: http://www.defra.gov.uk/foodfarm/farmanimal/diseases/atoz/ zoonoses/documents/reports/zoonoses2006.pdf

4. Tissot-Dupont $H$, Amadei MA, Nezri M, Raoult D. Wind in November, Q fever in December. Emerg Infect Dis. 2004;10(7):1264-9.

5. Hawker JI, Ayres JG, Blair I, Evans MR, Smith DL, Smith EG, et al. A large outbreak of $Q$ fever in the West Midlands: windborne spread into a metropolitan area? Commun Dis Public Health. 1998;1(3):180-7.

6. Carrieri MP, Tissot-Dupont H, Rey D, Brousse P, Renard H, Obadia Y, et al. Investigation of a slaughterhouse-related outbreak of Q fever in the French Alps. Eur J Clin Microbiol Infect Dis. 2002;21(1):17-21.

7. Gilsdorf A, Kroh C, Grimm S, Jensen E, Wagner-Wiening C, Alpers K. Large Q fever outbreak due to sheep farming near residential areas, Germany, 2005. Epidemiol Infect. 2008;136(8):1084-7.

8. Hawker J, Begg N, Blair I, Reintjes R, Weinberg J. Communicable disease control handbook. 2nd ed. Oxford: Blackwell publishing; 2006. p. 181.

9. Parker NR, Barralet JH, Bell AM. Q fever. Lancet. 2006;367(9511):679-88.

10. Carcopino X, Raoult D, Bretelle F, Boubli L, Stein A. Q Fever during pregnancy: a cause of poor fetal and maternal outcome. Ann N Y Acad Sci. 2009;1166:79-89.

11. Pebody RG, Wall PG, Ryan MJ, Fairley C. Epidemiological features of Coxiella burnetii infection in England and Wales: 1984 to 1994. Commun Dis Rep CDR Rev. 1996;6(9):R128-32.

12. Orr HJ, Christensen H, Smyth B, Dance DA, Carrington D, Paul I, et al. Case-control study for risk factors for $Q$ fever in southwest England and Northern Ireland. Euro Surveill. 2006;11(10). pii=655. Available from: http://www. eurosurveillance.org/ViewArticle.aspx?Articleld=655 
13. Jones A, Thomson D, Hort M, Devenish BJ. Met Office's nextgeneration atmospheric dispersion model, NAME III. Borrego $\mathrm{C}$, Norman $\mathrm{A}-\mathrm{L}$, editors. Air pollution monitoring and its application. Springer; 2007, p. 580-9.

14. Davies T, Cullen MJ, Malcolm AJ, Mawson MH, Staniforth A, White AA, et al. A new dynamical core for the Met Office's global and regional modelling of the atmosphere. Q J Roy Meteor Soc. 2005;131(608):1759-82.

15. Raoult D, Marrie T, Mege J. Natural history and pathophysiology of Q fever. Lancet Infect Dis. 2005;5(4):219-26.

16. Reid A, Malone J. Q fever in Ireland. A seroprevalence study of exposure to Coxiella burnettii among Department of Agriculture workers. Occup Med (Lond). 2004;54(8):544-7.

17. Starnini G, Caccamo F, Farchi F, Babudieri S, Brunetti B, Rezza G. An outbreak of $Q$ fever in a prison in Italy. Epidemiol Infect. 2005;133(2):377-80.

18. Gloster J, Sellers RF, Webster H, Valarcher J-F. Assessing the risk of airborne spread of foot-and-mouth disease: a case study. Weather. 2006;61:137-42.

19. Gloster J, Burgin L, Witham C, Athanassiadou M, Mellor PS. Bluetongue in the United Kingdom and northern Europe in 2007 and key issues for 2008. Vet Rec. 2008;162(10):298-302.

20. Veterinary Laboratories Agency, Health Protection Agency, Defra, Health and Safety Executive. Q fever: information for farmers. 2007: Available from: http://www.hpa.org.uk/web/ HPAwebFile/HPAweb_C/1210834106356. 